\title{
Epidemiological study in Okinawa, Japan, of human papillomavirus infection of the uterine cervix
}

\author{
TOSHIYUKI MAEHAMA \\ Department of Obstetrics and Gynecology, Faculty of Medicine, University of the Ryukyus, Okinawa, Fapan
}

(Received 16 fune 2004; accepted 14 September 2004)

\begin{abstract}
Objective. To investigate the prevalence and type distribution of human papillomavirus (HPV) in women with normal cervical cytology and with cervical intraepithelial neoplasia I to III(CIN) or carcinoma of the cervix in Okinawa, Japan.

Methods. We investigated HPV DNA in 4,078 subjects with cytologically normal cervices, 279 subjects with CIN, and 383 subjects with cervical cancer in Okinawa Prefecture in Japan. The presence of HPV DNA was also compared among generations. HPV DNA was both detected and typed using polymerase chain reaction (PCR).

Results. The HPV positivity rate was $10.6 \%$ in the subjects who were normal on cervical cytodiagnosis. In each generation, the positivity rate was $20.4 \%$ in women aged $20-29$ years and approximately $10 \%$ in the groups aged 30-89 years, with significant differences among generations. The HPV positivity rates in CIN and cervical cancer groups were $76.0 \%$ and $86.2 \%$, respectively, with no significant difference between the groups. The positivity rate of HPV 16 decreased with age in both CIN and cervical cancer groups.

Conclusion. Among non-cancer subjects, HPV infection rates were almost $20 \%$ in women aged $20-29$ years and $10 \%$ in women aged 30-89 years. HPV16-positive CIN or carcinoma were more prevalent in the younger women, suggesting that HPV16-infected epithelial cells rapidly progress to cervical cancer.
\end{abstract}

Keywords: Prevalence, cervical lesion, virus infection

\section{Introduction}

Although it is obvious that HPV is closely involved in the development of uterine cervical cancer, the reasons for onset of cervical cancer are not well understood. There have been only a few reports of the HPV prevalence rate in the uterine cervix in healthy women in Japan. A documentation of the prevalence might help in identifying the early phases of the disease, as well as contributing to an understanding of the natural history of HPV infection. Such information could be important in preventing the HPV infection and its consequences.

Several types of HPV associated with anogenital lesions have been identified in cervical specimens. These types, such as $16,18,31,33,35$ and 58 , are referred to as cervical carcinoma-associated HPVs.

In Okinawa Prefecture, the prevalence rate of cervical cancer is the highest in Japan. The prefecture is located about $1,500 \mathrm{~km}$ from the main islands of
Japan, and is a relatively closed colony with little population exchange with the main Japanese islands. Here we performed a large-scale epidemiological survey in which both the prevalence rate and the types of HPV were investigated. Healthy women and women with CIN or cervical cancer were included in this study.

\section{Materials and methods}

The study population was selected from women who underwent cervical cancer screening between 1994 and 1995, 4,078 subjects were normal on cervical cytodiagnosis. Additional subjects included 279 women with CIN and 383 women with cervical cancer, who visited the obstetrics and gynecology (OB-GY) outpatient department of Ryukyu University Hospital between January 1993 and September 1998. Consent was obtained for HPV testing. For HPV DNA detection, DNA was extracted from cells

Correspondence: T. Maehama, Department of Obstetrics and Gynecology, Faculty of Medicine, University of the Ryukyus, 207 Uehara, Nishihara-cho, Okinawa, T903-0215, Japan. Tel: 098-895-1177. Fax: 098-895-1426. E-mail: b983975@med.u-ryukyu.ac.jp

ISSN 1064-7449 print/ISSN 1098-0997 online (C) 2005 Taylor \& Francis Group Ltd DOI: $10.1080 / 10647440400028151$ 
collected on cervical swabs and amplified by PCR using HPVL1 consensus primers [1]. The HPV DNA was typed using type-specific primers $(16,18$, $31,33,35,58)$ [2]. Undetermined types were referred to as HPV $\mathrm{X}$.

\section{Results}

The HPV positivity rate was $10.6 \%$ in the subjects who were normal on cytodiagnosis. When analyzed by generation, the positivity rate peaked at a rate of $20.4 \%$ in the $20-29$ years age group, and persisted at a rate of about $10 \%$ after the age of 30 years up to 89 years (Table I). The rates were significantly different between the group aged 20-29 years and the group aged $30-39$ to $70-79$ years $(p<0.001)$. There was no significant difference between the groups aged 20-29 years and 80-89 years, because of the small number of cases. The frequencies of the high-risk HPV types $(16,18)$, intermediate-risk types $(31,33$, $35,58)$, and unknown (X) types were $0.3 \%(13 /$ $4,078), 1.3 \%(53 / 4,078)$, and $9.0 \%(368 / 4,078)$, respectively. As shown in Table I, type 35 was the most frequently detected known HPV type and type 18 was the least frequently detected type in the cytologically normal females.
The HPV positivity rate was $76.0 \%$ in CIN group, and the positivity rate by generation ranged from $61.5 \%$ to $88.9 \%$ (Table II). Among the HPV types, the frequency of type 16 was the highest at $19.3 \%$, and type 18 had the lowest frequency at $1.4 \%$ in the HPV-positive subjects. Type X accounted for $63.2 \%$. The type 16 positivity rate by generation was $40.6 \%$ in the $20-29$ and $20.7 \%$ to $6.3 \%$ in the $30-59$-yearold women, demonstrating a decrease with age.

The overall HPV positivity rate was $85.6 \%$ in the cervical cancer group, and the rate by generation ranged from $76.1 \%$ to $95.0 \%$ (Table III). Among the known HPV types, the $33.6 \%$ frequency of type 16 was the highest, followed by $7.3 \%$ for type $33,6.1 \%$ for type $31,5.8 \%$ for type $58,3.9 \%$ for type 18 and $2.7 \%$ for type 35 . The $40.6 \%$ frequency of type $\mathrm{X}$ was significantly lower than in the CIN group $(p<0.0001)$. The frequency of HPV type 16 decreased from 42.1 to $63.3 \%$ in the group who were $20-39$ years of age to $9.5 \%$ in the oldest group who were $80-89$ years of age.

\section{Discussion}

The mortality rate of cervical cancer has been decreasing, because of both the increased use of

Table I. HPV prevalence by age in cytologically normal cervical swabs.

\begin{tabular}{|c|c|c|c|c|c|c|c|c|c|}
\hline \multirow[b]{2}{*}{ Age (years) } & \multirow[b]{2}{*}{ No. of cases } & \multirow[b]{2}{*}{ No. HPV + (\%) } & \multicolumn{7}{|c|}{ HPV types $(\%)^{\star}$} \\
\hline & & & 16 & 18 & 31 & 33 & 35 & 58 & $\mathrm{X}$ \\
\hline $20-29$ & 275 & $56(20.4)$ & $3(5.4)$ & $0(0)$ & $4(7.1)$ & $0(0)$ & $4(7.1)$ & $0(0)$ & $48(85.7)$ \\
\hline 30-39 & 564 & $51(9.0)$ & $0(0)$ & $0(0)$ & $2(3.9)$ & $0(0)$ & $5(9.8)$ & $0(0)$ & $44(86.3)$ \\
\hline $40-49$ & 802 & $73(9.1)$ & $2(2.7)$ & $0(0)$ & $0(0)$ & $3(4.1)$ & $5(6.8)$ & $2(2.7)$ & $61(83.6)$ \\
\hline $50-59$ & 1039 & $104(10.0)$ & $2(1.9)$ & $1(1.0)$ & $2(1.9)$ & $3(2.9)$ & $4(3.8)$ & $2(1.9)$ & $90(86.5)$ \\
\hline $60-69$ & 1029 & $112(10.9)$ & $3(2.7)$ & $1(0.9)$ & $2(1.8)$ & $2(1.8)$ & $4(3.6)$ & $4(3.6)$ & $96(85.7)$ \\
\hline $70-79$ & 357 & $36(10.1)$ & $1(2.8)$ & $0(0)$ & $0(0)$ & $2(5.6)$ & $3(8.3)$ & $0(0)$ & $30(83.3)$ \\
\hline $80-89$ & 15 & $2(13.3)$ & $0(0)$ & $0(0)$ & $0(0)$ & $0(0)$ & $0(0)$ & $0(0)$ & $2(100)$ \\
\hline Total & 4078 & $434(10.6)$ & $11(2.5)$ & $2(0.5)$ & $10(2.3)$ & $10(2.3)$ & $25(5.8)$ & $8(1.8)$ & $368(84.8)$ \\
\hline
\end{tabular}

$\mathrm{X}, \mathrm{HPV}$ type not determined; ${ }^{\star}, \mathrm{HPV}$ type/HPV(+) in each generation.

Table II. HPV prevalence by age in CIN I-III.

\begin{tabular}{|c|c|c|c|c|c|c|c|c|c|}
\hline \multirow[b]{2}{*}{ Age (years) } & \multirow[b]{2}{*}{ No. of cases } & \multirow[b]{2}{*}{ No. HPV + (\%) } & \multicolumn{7}{|c|}{ HPV types $(\%)^{\star}$} \\
\hline & & & 16 & 18 & 31 & 33 & 35 & 58 & $\mathrm{X}$ \\
\hline $20-29$ & 39 & $32(82.1)$ & $13(40.6)$ & $0(0)$ & $1(3.1)$ & $1(3.1)$ & $0(0)$ & $0(0)$ & $17(53.1)$ \\
\hline $30-39$ & 106 & $82(77.4)$ & $17(20.7)$ & $2(2.4)$ & 7 (8.5) & $2(2.4)$ & $0(0)$ & $4(4.9)$ & $50(61.0)$ \\
\hline $40-49$ & 71 & $53(74.6)$ & $9(17.0)$ & $1(1.9)$ & $2(3.8)$ & $3(5.7)$ & $3(5.7)$ & $2(3.8)$ & $33(62.3)$ \\
\hline $50-59$ & 26 & $16(61.5)$ & $1(6.3)$ & $0(0)$ & $0(0)$ & $0(0)$ & $1(6.3)$ & $1(6.3)$ & $13(81.3)$ \\
\hline $60-69$ & 18 & $16(88.9)$ & $0(0)$ & $0(0)$ & $0(0)$ & $0(0)$ & $0(0)$ & $0(0)$ & $16(100)$ \\
\hline $70-79$ & 16 & $10(62.5)$ & $1(10.0)$ & $0(0)$ & $2(20.0)$ & $2(20.0)$ & $0(0)$ & $0(0)$ & $5(50.0)$ \\
\hline $80-89$ & 3 & $3(100)$ & $0(0)$ & $0(0)$ & $1(33.3)$ & $1(33.3)$ & $1(33.3)$ & $0(0)$ & $0(0)$ \\
\hline Total & 279 & $212(76.0)$ & $41(19.3)$ & $3(1.4)$ & $13(6.1)$ & $9(4.2)$ & $5(2.4)$ & $7(3.3)$ & $134(63.2)$ \\
\hline
\end{tabular}

$\mathrm{X}, \mathrm{HPV}$ type not determined; *, HPV type/HPV(+) in each generation. 
Table III. HPV prevalence by age in cervical cancer.

\begin{tabular}{|c|c|c|c|c|c|c|c|c|c|}
\hline \multirow[b]{2}{*}{ Age (years) } & \multirow[b]{2}{*}{ No. of cases } & \multirow[b]{2}{*}{ No. HPV + (\%) } & \multicolumn{7}{|c|}{ HPV types $(\%)^{\star}$} \\
\hline & & & 16 & 18 & 31 & 33 & 35 & 58 & $\mathrm{X}$ \\
\hline $20-29$ & 20 & $19(95.0)$ & $8(42.1)$ & $3(15.8)$ & $1(5.3)$ & $3(15.8)$ & $0(0)$ & $1(5.3)$ & $3(15.8)$ \\
\hline $30-39$ & 67 & $60(89.6)$ & $38(63.3)$ & $3(5.0)$ & $2(3.3)$ & $3(5.0)$ & $4(6.7)$ & $6(10.0)$ & $4(6.7)$ \\
\hline $40-49$ & 86 & $73(84.9)$ & $27(37.0)$ & $4(54.8)$ & $4(54.8)$ & $2(2.7)$ & $2(2.7)$ & $1(1.4)$ & $33(45.2)$ \\
\hline $50-59$ & 71 & $54(76.1)$ & $19(35.2)$ & $0(0)$ & $3(5.6)$ & $3(5.6)$ & $0(0)$ & $4(7.4)$ & $25(46.3)$ \\
\hline $60-69$ & 78 & $68(87.1)$ & $14(20.6)$ & $3(4.4)$ & $5(7.4)$ & $11(16.2)$ & $3(4.4)$ & $3(4.4)$ & $29(42.6)$ \\
\hline $70-79$ & 38 & $35(92.1)$ & $3(8.6)$ & $0(0)$ & $2(5.7)$ & $2(5.7)$ & $0(0)$ & $2(5.7)$ & $26(74.3)$ \\
\hline $80-89$ & 23 & $21(91.3)$ & $2(9.5)$ & $0(0)$ & $3(14.3)$ & $0(0)$ & $0(0)$ & $2(9.5)$ & $14(66.7)$ \\
\hline Total & 383 & $330(86.2)$ & $111(33.6)$ & $13(3.9)$ & $20(6.1)$ & $24(7.3)$ & $9(2.7)$ & $19(5.8)$ & $134(40.6)$ \\
\hline
\end{tabular}

$\mathrm{X}, \mathrm{HPV}$ type not determined; *, HPV type/HPV(+) in each generation.

mass examinations and the implementation of effective therapies. However, despite these advances, about 5,000 women die yearly in Japan of cervical cancer. Cervical cancer remains an important gynecological problem. Since Zur Hausen's group [3] detected HPV in cervical cancer tissues in 1983, it has become well recognized that HPV is the major risk factor for the development of cervical cancer. Much basic information about HPV and many clinical studies have been reported in the literature [4-6]. However, neither defined preventive measures nor therapeutic methods targeting HPV have been established. Since the mechanism of carcinogenesis after HPV infection has not been fully elucidated, it is very important epidemiologically to survey the HPV prevalence rate and types in the normal uterine cervix, in CIN and in cervical cancer in each generation.

In this study, on analysis of HPV positivity in cervical cytology-normal women by age, the positivity rate peaked to $20.4 \%$ in the group aged $20-29$ years, and persisted at about $10 \%$ in the groups aged $30-39$ to $80-89$ years. This finding was significant because it showed that infection occurred in young women aged 20-29 years at a rate which decreased by about half at ages 30-39 years, probably by elimination of unknown immunological mechanisms, and then persisted in women up to the age of 80-89 years. Therefore, although the prevalence of cervical cancer decreased in the group aged 80-90 years, HPV-positive individuals had the risk of developing cervical cancer.

On analysis of the frequencies of the HPV types in the subjects who were normal on cervical cytodiagnosis, type $\mathrm{X}$ dominated and accounted for $84.8 \%$, suggesting that the change from infection to malignancy might be infrequent. Melkert et al. [7] reported that the positivity rate was $10.7 \%$ in 4,146 subjects who had a normal cervical cytodiagnosis. The rates of $13.9 \%$ in subjects aged $15-34$ years and $6.6 \%$ in subjects $35-55$ years old were significantly different, suggesting that juvenile HPV infection was transient. Devilliers et al. [8] also reported that the positivity rate peaked to $17.8 \%$ in individuals aged $20-24$ years, and fell to $13.3 \%$ in subjects aged 25 29 years, $10 \%$ in subjects aged $30-49$ years, $6.1 \%$ in subjects aged $50-54$ years and $3.0 \%$ in $55-80$-yearold subjects. They hypothesized that the differences in the positivity rates among the various age groups were related to the extent of sexual activity, presumably higher in the younger individuals.

The HPV positivity rates of CIN and cervical cancer in this study were similar to rates reported in other studies [9]. About 90 types of HPV have been identified to date. The frequency of type 16 was the highest and type 18 was lowest among the HPV types detected in CIN. It is acknowledged that detection of HPV18 infections in CIN is difficult because of the rapid progress of the condition, and this may be reflected in the low frequency of detection. In cervical cancer, type 16 was the most frequent (33.6\%), type 35 was the least frequent $(2.7 \%)$ and type 18 frequency was $3.9 \%$. These findings in cervical cancer were similar to those of other studies of patients in Japan. However, the frequencies of types 16 and 18 in the USA were $47.1 \%$ and $23.5 \%$, respectively [10]. According to data collected from 22 different countries by Bosch and colleagues [11], detection rates for type 16 and type 18 were $50 \%$ and $14 \%$, respectively. Therefore, the positivity rates by type differed from those in this study, suggesting that geographic, racial and environmental factors are related to the prevalence of HPV types. The positivity rate of type 16 was the highest in CIN and cervical cancer. The type 16 positivity rate by generation decreased with age in both CIN and cervical cancer groups. These findings suggested that the relatively rapid progress of the cervical lesion after infection was due to the highrisk type 16. Therefore, HPV 16-positive young women with CIN should be carefully managed. 
Cervical cancer begins with HPV infection of the uterine cervix. Elucidation of the mechanism by which HPV progresses from infection to cervical cancer is necessary for the prevention of infection and effective therapeutic intervention after infection.

\section{References}

1. Yoshikawa $H$, Kawana $T$, Kitagawa $K$, Mizuno $M$, Yoshikura H, Iwamoto A. Detection and typing of multiple genital human papillomaviruses by DNA amplification with consensus primer. Jpn J Cancer Res 1991;82:524-531.

2. Asato T, Nakajima Y, Nagamine M, Nakashima Y, Takei H, Maehama T. Correlation between the progression of cervical dysplasia and the prevalence of human papillomavirus. J Infect Dis 1994;169:940-941.

3. Durst $M$, Gissman L, Ikenberg H, zur Hausen H. A papillomavirus DNA from a cervical carcinoma and its prevalence in cancer biopsy samples from different geographic regions. Proc Natl Acad Sci USA 1983;80:38123815.

4. zur Hausen H. Papillomaviruses in anogenital cancer as a model to understand the role of viruses in human cancers. Cancer Res 1989;49:4677-4681.

5. Herckenrode C, Malcolm A, Coleman D. Prevalence of human papillomavirus (HPV) infection in Basque country women using slot-blot hybridization: a survey of women at low risk of developing cervical cancer. Int $\mathrm{J}$ Cancer 1992;51:581-586.
6. Woodman C, Collins S, Winter H, Baily A, Ellis J, Prior P, Natural history of cervical human papillomavirus infection in young women: a longitudinal cohort study. Lancet 2001;357:1831-1836.

7. Melkert PWJ, Hopman E, Van Den Blule AJC, Risse EKJ, Van Diest PJ, Bleker OP. Prevalence of HPV on cytomorphologically normal cervical smears, as determined by the polymerase chain reaction, is age-dependent. Int J Cancer 1993;53:919-923.

8. De Villiers EM, Wagner D, Schneider A, et al. Human papillomavirus DNA in women without and with cytological abnormalities: results of a 5-year follow-up study. Gynecol Oncol 1992;44:33-39.

9. Sasagawa T, Dong $Y$, Saijoh K, Satake S, Tateno M, Inoue M. Human papillomavirus infection and risk determinants for squamous intraepithelial lesion and cervical cancer in Japan. Jpn J Cancer Res 1997;88:376-384.

10. Lorincz AT, Reid R, Jenson AB, Greenberg MD, Lancester W, Kurman RJ. Human papillomavirus infection of the cervix: relative risk associations of 15 common anogenital types. Obstet Gynecol 1992;79:328-337.

11. Bosch FX, Manos MM, Munoz N, et al. Prevalence of human papillomavirus in cervical cancer: a worldwide perspective. J Natl Cancer Inst 1995;78:796-802. 


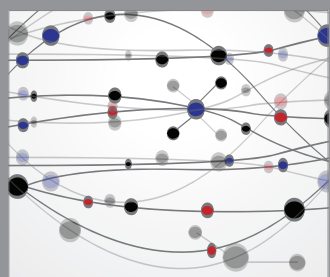

The Scientific World Journal
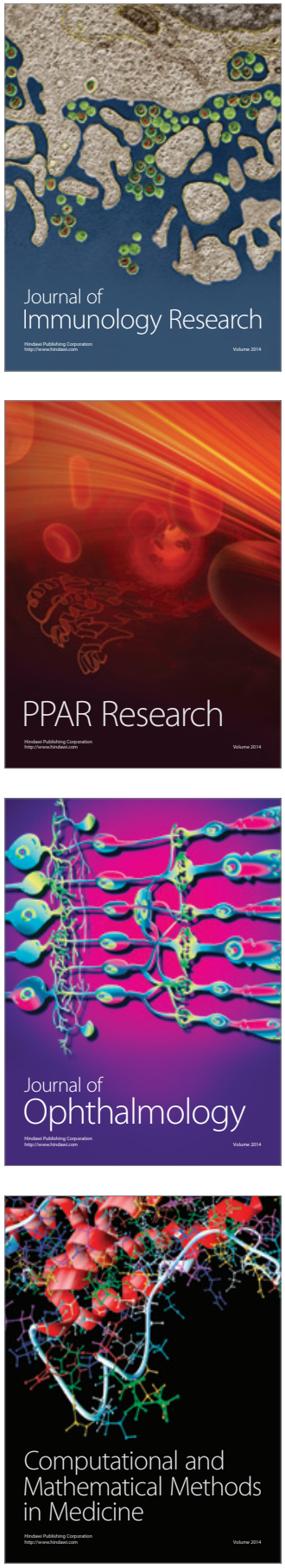

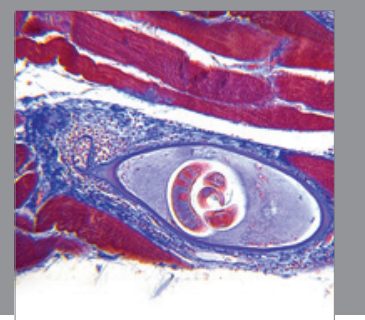

Gastroenterology

Research and Practice
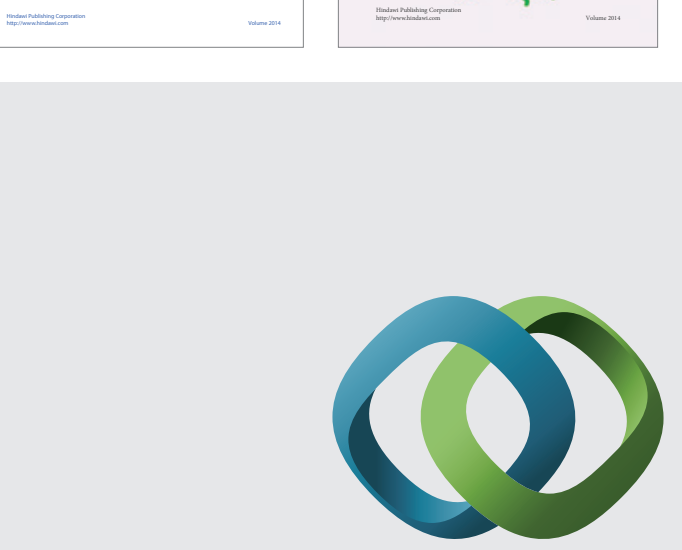

\section{Hindawi}

Submit your manuscripts at

http://www.hindawi.com
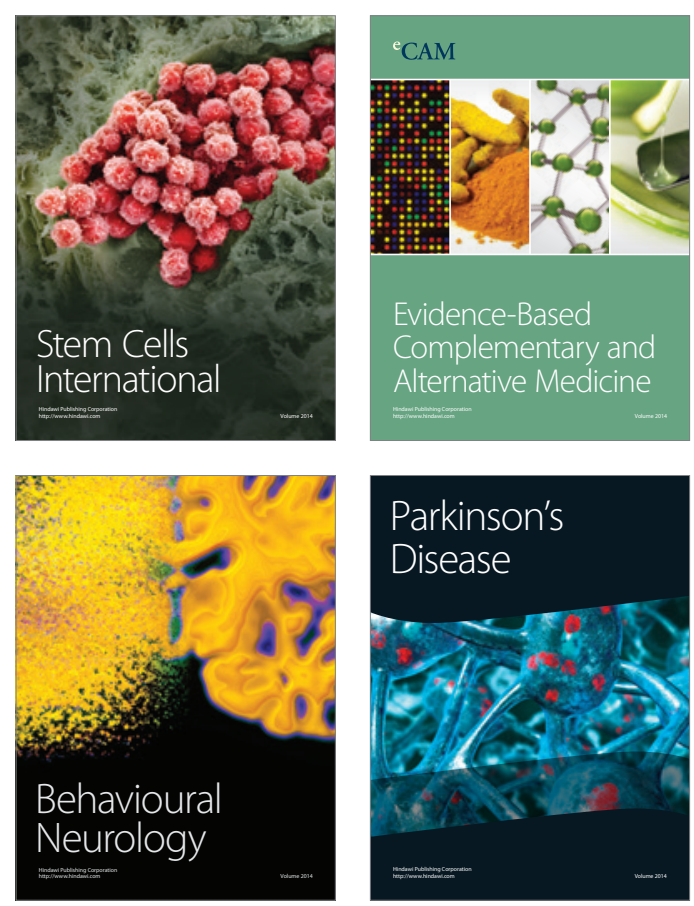

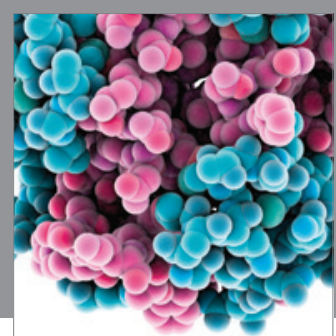

Journal of
Diabetes Research

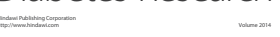

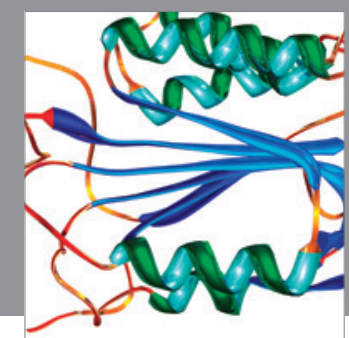

Disease Markers
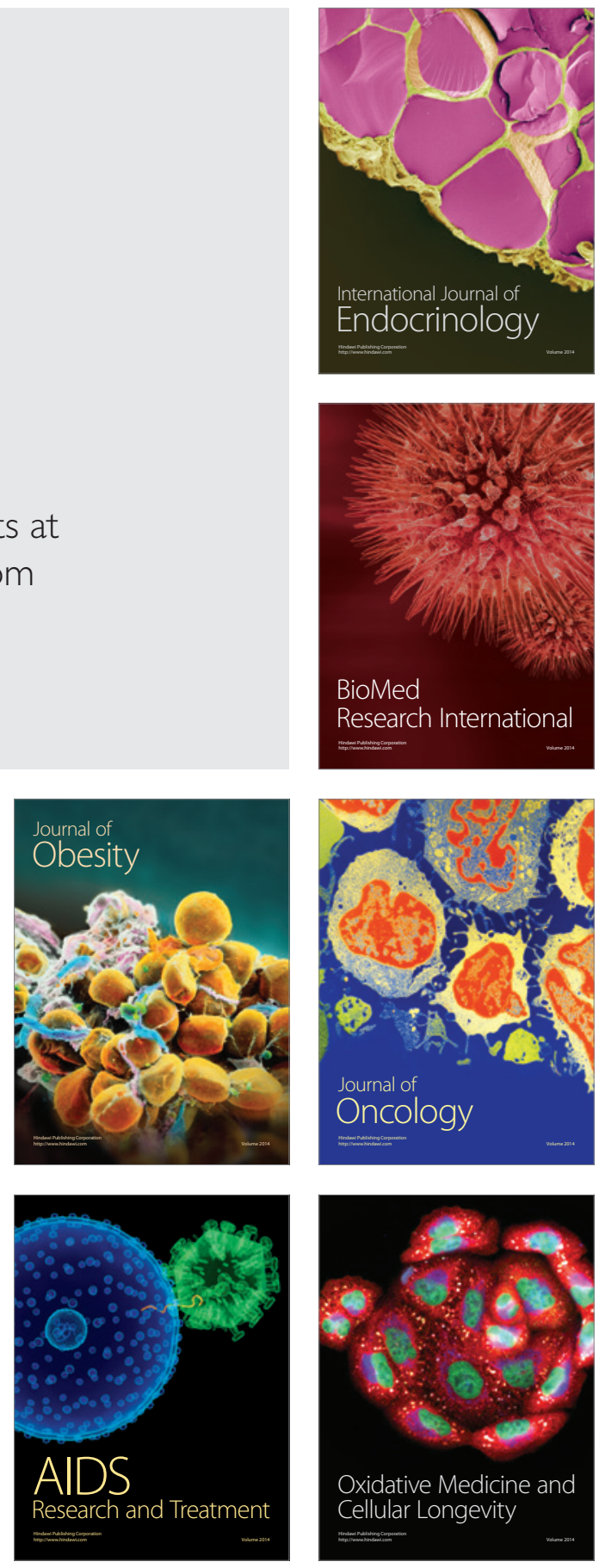